\title{
Zukünftiges Zukunftswissen in modernen Utopien: Bernhard Kellermann, Karl Ettlinger, Franz Werfel und Alban Nicolai Herbst
}

Spätestens seit dem 18. Jahrhundert sind Utopien in die Zukunft verlagerte alternative Gesellschaftsformen. ${ }^{1}$ So markiert für Reinhart Koselleck Louis-Sébastien Merciers im Jahr 1771 erschienener Roman L'An 2440, rêve s'il en fut jamais (1771) den „Einbruch der Zukunft in die Utopie“, d.h. die „Einverleibung der Utopie in die Geschichtsphilosophie“. (Koselleck 1982, 1) Als Rousseau-Schüler habe Mercier, so Koselleck, den Begriff der perfectibilité optimistisch gedeutet und in eine Fortschrittsgeschichte eingebettet. Das Narrativ ,Fortschritt‘ leistet demnach eine qualitative Differenzierung von Vergangenheit, Gegenwart und Zukunft und markiert ein zentrales Organisationsprinzip der diesem Narrativ folgenden utopischen Texte.

Wie sich hier zeigt, geht es in Utopien nicht einfach um die Darstellung einer zukünftigen Gesellschaft, vielmehr verhandeln Utopien stets auch gegenwärtige Zukunftskonzepte. Dies geschieht nicht nur durch die narrative Ausarbeitung der Folgen eines künftigen gesellschaftlichen Fortschritts, Niedergangs oder gar Zusammenbruchs, sondern auch über die Darstellung und Reflexion zukünftigen Zukunftswissens - schließlich muss man sich auch in der Zukunft zur Zukunft verhalten. Der zukünftigen Modellierung von Zukunft kommt hierbei eine herausragende Rolle $\mathrm{zu}$, weil mit ihr utopische Texte erstens ihre eigenen Bedingungen thematisieren, sie zweitens gegenwärtige futurische Aussagen, Verfahren und Diskurse reflektieren, und sie drittens mögliche, heute noch nicht realisierte Formen der Prognose entwerfen. ${ }^{2}$

Im Folgenden werde ich am Beispiel von vier Utopien - Bernhard Kellermanns Der Tunnel (1913), Karl Ettlingers Der erschossene Storch (1930), Franz Werfels Stern der Ungeborenen (1946) und Alban Nicolai Herbsts Trilogie Anders-

1 Im Folgenden fasse ich unter den Begriff, Utopie‘ auch Anti-Utopien und Dystopien, zumal die Wende zur „dark side“ der Utopie, wie Fátima Vieira $(2010,15)$ ausführt, auf die Ausrichtung der Utopie auf die Zukunft und auf die Idee wissenschaftlichen und technischen Fortschritts bezogen ist.

2 Utopien sind damit Teil prognostischer Dispositive und zugleich eine Reflexionsform dieser Dispositive. Zu einer kulturellen Prognostik vgl. Bühler und Willer 2016. 
welt $(1994,1998,2013)$ - untersuchen, wie Zukunftswissen in Utopien verhandelt wird. Dabei wird hier gerade nicht eine Differenz zwischen jüdischen und nichtjüdischen Utopien behauptet, vielmehr zielen die folgenden Ausführungen darauf ab, das breite Spektrum von Erscheinungsformen des Zukunftswissens in Zukunftsromanen in der Literatur des 20. Jahrhunderts aufzuzeigen. Der literaturgeschichtliche Wandel der Utopie im 20. Jahrhundert kann in diesem Rahmen nur angedeutet werden. Doch anhand der ausgewählten Romane werden historische Zäsuren durchaus deutlich: Während Kellermanns Roman der Technikeuphorie des frühen 20. Jahrhunderts zuzuordnen ist, thematisiert Ettlinger implizit die politische und ökonomische Krisensituation um 1930. Werfels Reflexionen von Zukunft sind vor dem Hintergrund des Zweiten Weltkrieges und der Shoah zu lesen, und Herbsts Trilogie knüpft an aktuelle Diskussionen um Klimawandel, Sicherheit oder neue Medien an. An den vier Romanen zeigen sich aber auch unterschiedliche Erzählorganisationen. Im Gegensatz zur konventionellen, linearen Erzählweise bei Kellermann und Storch bieten die anderen beiden Romane weitaus reflektiertere Erzählstrategien, wobei Herbsts postmoderne Romane die Selbstreferentialität des Textes auf die Spitze treiben. Bevor ich auf die einzelnen Romane eingehe, seien Formen der Zukünftigkeit von Utopien näher erläutert.

\section{Formen der Zukünftigkeit in utopischen Romanen}

Der ,Fortschritt‘ markiert das Basis-Narrativ und Organisationsprinzip utopischer Texte vom 18. bis 21. Jahrhundert. Dabei erscheint ,Fortschritt‘ in einer großen Bandbreite: Man konzipiert ihn als perfectibilité oder nach sozialistischen oder (pseudo-)evolutionistischen Modellen, sieht in ihm den Weg zu einer ,besseren` Gesellschaft oder aber einen Irrweg, der bis zum Untergang der menschlichen Zivilisation führen kann. Die Utopie avanciert somit zu einer zentralen Gattung der Moderne, denn sie widmet sich den möglichen ethischen, sozialen und politischen Folgen von Fortschritts-Prozessen. Auch die Entwürfe alternativer Gesellschafts-Modelle sind genuiner Teil der Moderne, denn als Reaktion auf die Modernisierung fordern Werke wie William Morris' News from Nowhere (1890) oder Carl Amerys Der Untergang der Stadt Passau (1975) eine Rückkehr zu einfachen und möglichst natürlichen Lebensweisen.

Am Narrativ des Fortschritts zeigt sich aber auch das ambivalente Verhältnis der Utopie zur Moderne. Zu Beginn des 20. Jahrhunderts haben technokratische Utopien große Konjunktur, man denke an H.G. Wells' Anticipations of the Reaction of Mechanical and Scientific Progress Upon Human Life and Thought (1901), Theodor Herzls Sozial-Utopie Altneuland (1902), Bernhard Kellermanns Technik-Utopie Der Tunnel (1913) oder das Projekt Atlantropa (1932) des Architekten Herman 
Soergel. Allerdings wird zunehmend auch der Fortschritt selbst problematisiert, womit die Utopie zur Dystopie wird. Aldous Huxley führt die fortschrittsskeptische Perspektive im Vorwort seines dystopischen Romans Brave New World (1932) folgendermaßen aus:

The theme of Brave New World is not the advancement of science as such; it is the advancement of science as it affects human individuals [...]. The only scientific advances to be specifically described are those involving the application to human beings of the results of future research in biology, physiology and psychology. (Huxley 1977, 10)

Die zukünftigen Anwendungen zukünftiger Forschungen und deren Folgen auf das soziale Gefüge dekliniert Huxley anhand der behavioristischen Organisation der Gesellschaft durch. Huxleys Selbstbeschreibung seines Romans gilt ebenso für andere Werke dieses Genres, George Orwells Roman 1984 (1949) zum Beispiel entfaltet die zukünftigen Möglichkeiten der Überwachungstechnik und ihre Folgen für die Organisation der Gesellschaft.

Dementsprechend greifen Dystopien seit dem späten 19. Jahrhundert gegenwärtige Themen wie Industrialisierung, nuklearer Krieg, Bevölkerungsexplosion oder Klimawandel auf und verlagern sie in die Zukunft. Arno Schmidts Dystopie Schwarze Spiegel (1951) zum Beispiel schildert, wie das Leben eines ,letzten Menschen' nach einem Krieg mit Nuklearwaffen aussehen könnte. Radikaler als Schmidts bürgerliches Szenario verfährt Cormack McCarthys Roman The Road (2006), der wie in einem Gedankenexperiment die sozialen und ethischen Folgen eines nuklearen Winters, in dem es keine Nahrungsmittel mehr gibt, durchspielt. ${ }^{3}$

Auch auf der diskursiven Ebene sind Utopien als in die Zukunft verlagerte Reflexionen der Gegenwart zu verstehen. Wenn der Protagonist in H.G. Wells' The Time Machine (1895) darüber räsoniert, ob die Zweiteilung der Arten in Eloi und Morlocks aus der Kluft zwischen Kapitalisten und Arbeitern oder der Anpassung an die Umweltbedingungen resultiert, erklärt er die zukünftige Gesellschaftsordnung auf der Grundlage der gegen Ende des 19. Jahrhunderts dominanten sozialistischen und evolutionistischen Konzepte. (Vgl. Willer 2012) Auch Konflikte über die Ausrichtung des Sozialismus werden über Zukunftsromane ausgetragen, so führte William Morris seine Kritik am Technizismus von Edwards Bellamys Roman Looking Backward (1888) nicht nur in einer Rezension aus, sondern auch mit seiner Utopie News from Nowhere (1890), die im Gegensatz zu Bellamys Roman eine naturnahe Agrar-Gesellschaft entwirft.

3 Zu McCarthy und allgemein zu aktuellen Entwürfen von „Zukunft als Katastrophe“ vgl. Horn 2014. 
Für den Literaturwissenschaftler Darko Suvin zeigt sich gerade am Beispiel von Bellamy und Morris, dass sich eine Gattung wie Science Fiction nicht über den Begriff Extrapolation definieren lässt. Bellamy und Morris nutzen nach Suvin den Kunstgriff der Antizipation, um sich mit den „im Entstehen begriffenen Verhältnissen in den achtziger Jahren des 19. Jahrhunderts“ zu befassen. (Suvin 1979, 107) Der „Erkenntniswert“ jeder Science Fiction-Literatur liegt nach Suvin daher „eher im analogen Bezug zur Gegenwart des Autors statt in vereinzelten oder globalen Voraussagen“. (Suvin 1979, 109) Suvins Argument gilt überhaupt für Zukunftserzählungen: In der Regel geht es ihnen nicht um Vorhersagen der Zukunft, sondern um reflektierte Auseinandersetzungen mit ihrer Gegenwart. Zukunftserzählungen konstituieren hierbei eine besondere Beobachterposition, denn die Gegenwart erscheint in ihnen als Vergangenheit, wie Fredric Jameson mit Bezug auf Science Fiction-Literatur ausführt: „SF thus enacts and enables a structurally unique ,method' for apprehending the present as history.“ (Jameson 1982, 153)

An der Science Fiction-Literatur zeigt sich damit die Eindimensionalität des futurologischen Verfahrens ,Extrapolation“. Denn die Zukunft ist, so Suvin, ein „Knoten vielfältiger Entwicklungen“ und bestimmt durch „Absichten, Wünsche und Überzeugungen“, also kein quantitativ messbarer Raum. (Suvin 1979, 108) Science Fiction-Literatur ist aber nicht nur negativ auf wissenschaftliche Verfahren bezogen. Nach Umberto Eco präsentiert sich Science Fiction als „narratives Spiel auf dem Wesenskern jeder Wissenschaft [...], nämlich auf ihrem Konjekturverfahren“. (Eco 2002, 219) Das Verfahren der Konjektur verweist somit auf den wissenschaftlichen Kern der Science Fiction sowie auf den narrativen Kern der Wissenschaft. (Vgl. Macho/Wunschel 2004; Siebenpfeiffer 2016) Auch für Utopien gilt, dass sie nicht nur Zukünfte imaginieren, sondern ebenso Arten der Herstellung von Zukunft reflektieren, wobei sie sich im Gegensatz zur Science Fiction-Literatur nicht nur auf wissenschaftliche Verfahren beschränken.

Einmal finden sich in Zukunftsromanen futurische Aussageformen, die jedem literarischen Text zukommen, wie Eberhard Lämmert bereits in seinen Bauformen des Erzählens (1955) entlang der Unterscheidung von zukunftsgewissen und zukunfts-ungewissen Vorausdeutungen differenziert aufgezeigt hat. Abgesehen davon, dass jeder Text in irgendeiner Art und Weise Formen der Zukünftigkeit aufweist, kommt utopischen Texten auch immer wieder ein selbstreferentieller Charakter zu. Zum Beispiel thematisiert Marlen Haushofers Roman Die Wand (1963) ,Zukunft‘ nicht nur in Hinsicht auf das Überleben nach - wie zu vermuten ist - dem Einschlag einer Neutronenbombe, sondern auch durch die Erzähltechnik der Vorausdeutung: In der retrospektiven Erzählung nimmt die Erzählerin andeutend zukünftige Ereignisse vorweg. Immer wieder werden die erzählten Ereignisse unterbrochen von Bemerkungen darüber, dass ihr treuer Hund und ihr 
Stier gewaltsam zu Tode kommen würden - was dann auch geschieht. Während hier das angedeutete Ereignis eintritt, weist die geradezu leitmotivisch auftretende Frage nach der Fortsetzung ihrer eigenen Existenz sowie derjenigen der menschlichen Spezies über den Zeitpunkt des Erzählens hinaus. Dieser textimmanente, offene Zukunftshorizont rekurriert seinerseits, im Sinn einer mise en abyme, auf den gesamten Roman, welcher den gesellschaftlichen Zukunftshorizont in Form der Utopie nicht einschließt, sondern offen lässt: Es sind auch andere Erzählungen von der Zukunft möglich.

Utopien reflektieren Zukunftswissen aber auch anhand der Art und Weise, wie in der Zukunft die Zukunft konstruiert wird, und anhand des Verhältnisses der Bewohner der utopischen Welt zu ihrer Zukunft. Konflikte entstehen in utopischen Erzählungen immer wieder gerade dadurch, dass die Bewohner der zukünftigen Welt unterschiedliche Vorstellungen über die Zukunft haben. In Herzls Altneuland (1902) kommt David Littwak mit seinen Freunden in die Ortschaft Neudorf, wo Dissens über die zukünftige Zuwanderung von Nichtjuden besteht. (Vgl. Herzl 1978 [1902], 98) Um die Dorfbewohner für sich zu gewinnen, rekurriert Littwak in seiner Rede auf die Modelle, auf denen Ortschaften wie Neudorf basierten, wie die Experimente der Pioniere von Rochdale, die im 19. Jahrhundert den ersten Genossenschaftsladen gründeten, oder sozialistische Utopien - von Fourier über Cabet bis Bellamy und Hertzka. (Vgl. Herzl 1978 [1902], 100-105) Demnach beruht die neue Gesellschaft auf den Ideen sämtlicher Kulturvölker, und so müsse man auch die aufnehmen, die diese Gesellschaftsordnung anerkennen. Hier zeigt sich auch die Verschachtelung unterschiedlicher Zukünfte: Um sein Zukunftsprojekt zu verwirklichen, greift der in der Zukunft lebende Littwak auf vergangene Zukunftsideen der Sozialisten zurück.

Wie damit deutlich wird, ist zukünftiges Zukunftswissen und -handeln keineswegs nur ein Motiv oder Erzählelement neben anderen: Die Fiktion einer zukünftigen Gesellschaft erlaubt vielmehr, unterschiedliche Formen des Zukunftswissens und -handelns auszuformulieren, zu kontrastieren und miteinander zu konfrontieren. Indem somit die utopischen Texte immer wieder an aktuell verhandelten Wissensformen wie der Evolutionstheorie, dem Behaviorismus oder der Ökologie ansetzen, sozialistische, zionistische, totalitäre oder neoliberale Gesellschaftsmodelle darstellen, neue mit alten Prognosetechniken verbinden, die ökonomischen, sozialen und politischen Dimensionen von Zukunftswissen vorführen, reflektieren sie die gegenwärtige Zukunft in der zukünftigen Zukunft und entfalten unterschiedliche Formen der Prognose. 


\section{Held der Zukunft: Bernhard Kellermanns Der Tunnel}

Bernhard Kellermanns im Jahr 1913 erschienener Roman Der Tunnel spielt in der nahen Zukunft, worauf einige Bemerkungen zu den technischen Möglichkeiten schließen lassen. Zum Beispiel ist die Rede von einem internationalen Rundflug um die Erde oder von zwei Luftschiffen, die den Atlantik überquert hätten (vgl. Kellermann 1985 [1913], 91 und 90) - tatsächlich gelang die erste Überquerung im Jahr 1919. Diese ,leichten` Verschiebungen in eine nahe Zukunft sind narrative Strategien, um dem eigentlichen Thema, dem Tunnelbau, Plausibilität zu verleihen: Schließlich gibt es 1913 bereits elektrische Autos in New York, und eine versuchte Atlantiküberquerung mit einem Luftschiff war erst im Jahr 1910 gescheitert. Dieses Erzählverfahren hat David Lodge bereits in George Orwells 1984 ausgemacht: Orwell imaginiere ,the future by invoking, modifying and recombining images of what his readers, consciously or unconsciously, already knew“. (Lodge 1992, 136-137)

Zwar liefert der Roman keine organisatorischen Details einer zukünftigen Gesellschaftsform, doch bringt er zum Ausdruck, die Umsetzung des Bauprojekts - der Ingenieur Mac Allen möchte einen Tunnel zwischen den USA und Europa bauen - werde den Weltverkehr und damit die wirtschaftliche und politische Ordnung nachhaltig verändern. Ein Hauptinvestor des Projekts formuliert es so: „Europa wird ein Vorort Amerikas werden.“ (59) Die Planung und der Bau eines solchen Tunnels lassen sich als Allegorie für eine im Jahr 1913 noch nicht realisierte, technokratisch organisierte Gesellschaft lesen. Deutlich wird das Vertrauen auf die Technik in der Vision einer zukünftigen anderen Stadt: Mac Allen wirbt für den Bau einer Stadt am Tunneleingang folgendermaßen:

\footnotetext{
Diese Plakate zeigten eine Feenstadt, einen Ozean von Häusern, aus der Vogelperspektive gesehen. Nie hatte ein Mensch etwas Ähnliches gesehen oder erträumt! In der Mitte dieser Stadt [...] lag eine grandiose Bahnhofsanlage, im Vergleich zu der Hudson-River-Terminal, Central- und Pennsylvania-Station Kinderspielzeuge waren. [...] Ein dichtgedrängtes Gewimmel tausendfenstriger Wolkenkratzer scharte sich um den Bahnhofsquare: Hotels, Kaufhäuser, Banken, Officebuildings, Boulevards, Avenuen, in denen die Menge wimmelte, Autos, elektrische Bahnen, Hochbahnen dahinschossen. Endlose Reihen von Häuserblocks, die sich im Dunst des Horizonts verloren. (92-93)
}

Die Beschreibung dieser zukünftigen Stadt folgt dem Prinzip der Übersteigerung gegenwärtig existierender Dinge: Zwar kennt man bereits Hochhäuser, aber noch kein Gewimmel von Wolkenkratzern. Diese Überschreitungslogik des Gegenwärtigen zeigt sich aber vor allem an der Metapher des Horizonts, der spätestens seit 
Francis Bacons berühmtem Titelkupfer seiner Instauratio Magna (1620) als Figur der Überschreitung des Bekannten und des Aufbruchs in eine unbekannte Welt fungiert, ob diese jenseits der Säulen des Herkules oder in der Zukunft liegt. Kellermanns Beschreibung verbindet diese räumliche Grenzüberschreitung mit einer zeitlichen Ausrichtung. Das Bild der „endlosen Reihen der Häuserblocks, die sich im Dunst des Horizonts verloren“, verweist auf den endlosen Fortschritt der Technik: Dieser Fortschritt wird im Roman nicht nur genannt, und es geht auch nicht alleine um das Ergebnis des Fortschritts, sondern der Tunnelbau sowie die Geschwindigkeit des Zuges führen den technischen Fortschritt, der auch die soziale Ordnung verändern wird, performativ vor.

Ausgehend von einer noch nahe an der Gegenwart liegenden Zukunft stellt der Roman damit die Herstellung der zukünftigen Welt dar. Was Kellermanns Roman am Beispiel des Tunnel-Projekts aber insbesondere vorführt, ist die Frage, wer überhaupt dazu autorisiert ist, über die Zukunft $\mathrm{zu}$ sprechen, und mit welchen Verfahren diese Autorität erlangt werden kann.

Der Ingenieur Mac Allen wird zu Beginn des Romans ausführlich charakterisiert: Er sitzt im ersten Kapitel mit seiner Frau bei einem Konzert, hört aber nicht der Musik zu, sondern misst „mit den Blicken die Dimensionen des ungeheuren Saales aus, dessen Decken- und Logenringkonstruktion er bewunderte“. Er schätzt die stündlichen Beleuchtungskosten ab und ist verblüfft über die „maschinelle Exaktheit, mit der das Orchester arbeitete“. (12) Mac Allen ist aber nicht nur ein Ingenieur, der seine Umwelt ausschließlich mit technischem und ökonomischem Interesse betrachtet, er wirkt auch als Person auf Menschen. Als er Baugrundstücke für seine zukünftige Stadt verkauft, um sie zu finanzieren, verstehen ihn auch die kleinen Leute, denn er „trieb keinen Humbug mit endgültig vergangenen und unkontrollierbaren zukünftigen Dingen, dieser Mann war die Gegenwart. Er versprach etwas Handgreifliches, das jeder verstehen konnte: Er wollte ein Loch durch die Erde graben, das war alles!“ (102)

Der Roman schildert somit die Geschichte, wie Mac Allen bei Investoren, Arbeitern und Anlegern zunehmend Vertrauen für sein Projekt gewinnt. Damit verbindet er zwei unterschiedliche Figuren miteinander: Mac Allen ist gleichzeitig Ingenieur und Projektemacher. Mit letzterem schließt Kellermanns Roman an eine Figur an, die bereits im 17. Jahrhundert als Typus von planvoller Zukunftsgestaltung bekannt oder besser gesagt: verrufen war. ${ }^{4}$ Denn Projektemacher haben zwar Pläne und Vorhaben, aber entweder lassen sich diese von vornehe-

4 Einen Überblick über die Figur des Projektemachers bietet der Sammelband von Krajewski 2004. 
rein nicht umsetzen oder sie scheitern während der Realisierung. Laut dem Verfasser des Eintrages „Projectenmacher“ in Zedlers Universallexikon müsse man ihnen nicht „sogleich Gehör geben, weil sie insgemein Betrüger sind“. (1741, Sp. 784) In Kellermanns Roman ist der Projektemacher allerdings ein Ingenieur, und als solcher Experte für die Realisierung von Plänen und Projekten. So heißt es über Mac Allen: „Das Projekt war sein Leben! Er rechnete seine Chancen durch wie ein algebraisches Problem, bei dem jedes einzelne Glied das Resultat der vorhergehenden Resultate ist.“ (24) Demgemäß wird er sein Projekt verwirklichen, aber das Scheitern spielt durchaus eine wichtige Rolle, denn von vornherein ist deutlich, dass Mac Allens Projekt Risiken aufweist. Insofern ist es nur konsequent, dass das Geld in Kellermanns Roman ebenso von Bedeutung ist wie die Technik: Nur wer das Risiko eingeht, Geld zu verlieren, wird auch Gewinne machen können. Parallel dazu gilt für die technische Seite: Nur wer bereit ist, Menschenleben aufs Spiel zu setzen, wird ein großtechnisches Projekt fertigstellen können.

Das Scheitern findet konkret in Gestalt eines technischen Unfalls statt, bei dem über dreitausend Arbeiter sterben, und einer finanziellen Katastrophe des Syndikats, das den Tunnelbau durch Aktien finanziert. Dieser doppelte Unfall unterbricht zwar die Heldengeschichte, aber nur, damit am Ende der Erfolg des Projekts umso glänzender erscheinen kann. Denn trotz aller persönlichen, technischen und ökonomischen Widrigkeiten gelingt es Mac Allen, den Tunnel zu bauen, worauf ihn die Welt als Helden bejubelt. Der Projektemacher ist also keine gescheiterte Existenz, sondern einer, der sich auch gegen Widerstände und Unfälle durchsetzt, weshalb Mac Allen für Segeberg die Verherrlichung der Technik verkörpert. (Vgl. Segeberg 1987, 206) ${ }^{5}$ Eine andere Lektüre hat dagegen Robert Leucht vorgeschlagen: Nach ihm analysiert der Roman den Typus des Ingenieurs kritisch, indem er „den Ingenieur als Helden nicht von Beginn an setzt, sondern vielmehr seine Verfertigung zu einem Helden rekonstruiert“. (Leucht 2011, 301) Die Darstellung der Genese des Helden schließt jedoch nicht seine Heroisierung aus.

Kellermanns Zukunftsroman stellt nicht nur eine zukünftige Gesellschaft dar, vielmehr zielt er auf die Schilderung der Realisierung eines technischen Projekts und damit auf die Entstehung einer neuen Gesellschaftsform in der nahen Zukunft. Damit führt er Konzepte von Zukunftswissen und -handeln vor und reflektiert die Frage der Autorisierung futurischer Sprechakte. Der Tunnelbau ist somit

5 Laut Segeberg finde mit der Glorifizierung aber auch der Übergang vom Ingenieur als dem „Auserwählten“ zum „Ingenieur des täglichen Lebens“ statt, d.h. die Erhöhung des Ingenieurs geht einher mit seiner Angleichung an den „soldatischen Destruktionstechniker“. 
auch als Referenz auf die technokratische Vision des Romans selbst zu lesen. Denn so wie Mac Allen den Bau trotz aller Hindernisse zu Ende bringt, wird sich auch, so wäre zu schließen, die Gesellschaft technisch weiterentwickeln - wenn sie denn Helden wie den Ingenieur Mac Allen hervorbringt.

\section{Die letzten Menschen: Karl Ettlingers Der erschossene Storch}

Karl Ettlinger (1882-1938) stammte aus einem jüdischen Frankfurter Bürgerhaus. Die Eltern betrieben eine Fabrik, wozu er aber, so Ettlinger selbst, so viel Talent gehabt habe wie die „Kuh zum Foxtrott“. (zit. nach Hock 1998, 31) ${ }^{6}$ Dafür fand er 1902 eine Anstellung bei der Wochenschrift Jugend in München, bei der er 25 Jahre lang blieb. Bekannt wurde Ettlinger vor allem durch seine Mundartdichtung, satirische Erzählungen, Parodien, Anekdoten-Sammlungen und Humoresken. Im Ersten Weltkrieg wurde er verwundet und erhielt das Eiserne Kreuz II. Klasse, in den 1920er Jahren arbeitete er als freier Schriftsteller. 1936 erhielt er absolutes Berufsverbot, konnte aber noch als Vortragender für den jüdischen Kulturbund arbeiten. Im Jahr 1938 reiste er nach Berlin, möglicherweise wollte er in die USA emigrieren, verstarb aber im Jüdischen Krankenhaus in Berlin bei einer Gallenoperation.

Der Roman Der erschossene Storch erschien im Jahr 1930 und kann, wie später noch dargelegt wird, geradezu als Konterpart zu Kellermanns Fortschritts-Utopie verstanden werden. Denn bei Ettlinger erwacht der Rechtsanwalt Dr. Jung nach einem fast fünftausend Jahre währenden Schlaf, um feststellen zu müssen, dass der letzte große Krieg nur zehn Minuten dauerte und danach nicht nur Jungs Heimatstadt Düsseldorf, sondern ganz Deutschland, England, Frankreich und Amerika nicht mehr existieren. Als Konsequenz verzichtet die Gesellschaft auf Nachkommen. Dr. Jung erwacht in einem Land, das keinen Namen hat, aber Land ohne Kinder genannt werden könne: Es ist das „Land, in dem es keine Kinder gibt. Und niemals mehr Kinder geben wird. Denn wir wollen glücklich sein“. (Ettlinger 1930, 17) Ganz richtig stellt Dr. Jung fest, dass die Menschheit sich damit selbst zum Aussterben verurteilt habe.

Dabei hat auch diese zukünftige Gesellschaft insbesondere technische Fortschritte hinter sich gebracht. Zum Beispiel verfügt sie über einen Armreif, der

6 Zu Ettlingers Lebensgeschichte vgl. Hock 1997. 
einen geräuschlosen Motor verbirgt, mit dem man fliegen kann, was für Dr. Jung die Sache noch unverständlicher macht:

Und alle diese kostbaren Funde des Menschengeistes sollen verloren gehen, durchrieselte es Dr. Jung. Alles was das Genie ersann und schuf, soll in dem Massengrab einer entvölkerten Erde verfaulen, nur weil dieses „Land ohne Kinder“ aussterben, oder wie sie es nennen, „glücklich sein“ will? ... (33)

Während bei Kellermann der Fortschritt trotz der unvermeidlichen Unfälle und Rückschläge eine bessere Welt bringt, führt er bei Ettlinger in einen grausamen Krieg. Kellermanns technokratische Vision erhält bei Ettlinger ihr Korrektiv: Der technische Fortschritt alleine - die Bewohner des Landes ohne Kinder können selbst die Jahreszeiten steuern und den Frühling zwanzig Jahre währen lassen (vgl. 44) - konstituiert eben noch keine stabile Gesellschaftsform. Die Konsequenz, die Ettlingers Protagonisten aus den Erfahrungen des Krieges ziehen, sind wohl singulär in seiner Zeit: Nämlich die absichtliche Auslöschung der Menschheit durch freiwilligen Verzicht auf Fortpflanzung.

Die Bewohner des Landes ohne Kinder sind somit eine Gemeinschaft der „letzten Menschen“. (91) Die Entscheidung, keine Kinder mehr zu bekommen, ist eine Konsequenz des letzten Krieges, der als Folge des Fortschritts erscheint und dessen Beschreibung an den Ersten Weltkrieg erinnert: Die Erde sei in Petroleum getränkt gewesen, die Luft durchzuckt von Giftgasen, und Helden habe es in diesem Krieg genauso wenig gegeben wie es im Schlachthaus Helden unter den Tieren gebe. (Vgl. 85-86) Die wenigen, die den Krieg überlebten, versammelten sich um den Sonderling und Wissenschaftler Cassius, der ausdrücklich nicht mit Noah zu identifizieren sei: Cassius habe das Unglück nicht vorhergesehen, sei nicht gewarnt worden und habe auch keine Vorbereitungen getroffen. (Vgl. 8889) Und wie seine Ansprache an die Überlebenden des Krieges verdeutlicht, ist Cassius eher als ein Anti-Noah zu verstehen: ${ }^{7}$

„Wollt ihr die Wiederholung der Greuel in noch furchtbareren Maßen?“ „Nie!“, tobten sie. „Dann hört auf euch zu vermehren! Jedes Kind, das ihr dem Licht schenkt, kann der Tausendste sein, der vom Gifte falschen Ehrgeizes Zerfressene, der die anderen Neunhundertneunundneunzig in das Sklavenjoch seiner weltumspannenden Pläne spannt, seiner Pläne, die sich von Strömen Menschenbluts ernähren! Frauen, Mädchen, wollt ihr Kinder in die Welt setzen, damit in der Blüte des Lebens ihre Leiber in Fetzen gerissen, ihre Augen von Gasen geblendet, ihre Glieder von Geschossen zerschmettert werden?“ (95)

7 Eine interessante aktuelle Parallele hierzu bietet Darren Aronofskys Film Noah (2014), in dem Noah die Fortexistenz der Menschheit verhindern will. 
Während Gott nach der Sintflut Noah und seine Söhne mit den Worten segnet „Seid fruchtbar und mehret euch und füllet die Erde“ (Mose 1, 9), preist Cassius das „Glück einer im Überfluß aussterbenden Menschheit“. (96) Für die Bewohner des „Landes ohne Kinder“ gibt es weder einen Gott noch Hoffnung auf eine zukünftige Welt.

Mit dem Auftauchen von Dr. Jung kommt dann allerdings eine zweite Perspektive ins Spiel. Denn natürlich ist der Düsseldorfer Anwalt äußerst erbost über das Aussterben der Menschheit, was er gerade mit Blick auf die Dimension der Zukunft verdeutlicht: Das Land ohne Kinder sei ein Gefängnis, in dem mit dem „Gesetz der Unfruchtbarkeit“ alle Zukunftsträume ausgelöscht worden seien. (145) Demgemäß arbeiten die Bewohner dieses Landes nicht, weder verbessern sie die bestehenden Einrichtungen, noch forschen sie. Vielmehr genießen sie verschwenderisch ihr Leben.

Ettlingers Zukunftsroman interessiert sich nicht für die Struktur der zukünftigen Gesellschaft, sondern für ihr Zukunftsverhältnis. Im Kontrast der beiden Aussichten, Aussterben der Menschheit oder Fortführung durch Nachkommen, verhandelt er die Zukunftsaussichten seiner eigenen Gegenwart - nach dem Ersten Weltkrieg und der Weltwirtschaftskrise. Damit hat auch Ettlinger, wie Kellermann, eine Mission. Natürlich wird am Ende eine junge Frau ein Kind gebären, die Frauen des Landes ohne Kinder werden in Verzückung geraten, und der Herrscher Cassius wird eine Kehrtwende machen: Seine Narrengesetze seien einem verfaulenden Baumstamm vergleichbar, bei dem sich keine Äste in den „Himmel der Zukunft“ ausbreiteten. (209) Allerdings folgt Cassius keineswegs Dr. Jungs hoffnungsvollem Zukunftsoptimismus, denn der Kampf sei aller „Lebenszweck und Inhalt“, wobei sein Endziel verborgen sei und nur geahnt, nicht aber gewusst werden könne. (210) Auf der Grundlage von Nietzsches Philosophie prophezeit Cassius denn auch am Ende, dass sich die Geschichte wiederholen werde, Zwietracht und Kämpfe würden wiederkehren. Der Mensch könne den Kampf nicht verhindern, nur „veredeln“, so dass man nicht mehr um Macht und Territorien, sondern um „Schätze des Geistes, der Idee, der Seele“ kämpfe. (212) Das Ende bleibt damit zwiespältig, zwar ist die Zeit der Unfruchtbarkeit vorüber, doch das nietzscheanische Bild der Wiederkehr des Gleichen relativiert die Aufbruchsstimmung der neuen Gesellschaft. 


\section{Zukunft erzählen: Franz Werfels Stern der Ungeborenen}

Auch in Franz Werfels Roman Stern der Ungeborenen $(1946)^{8}$ finden sich in der Zukunft unterschiedliche Positionen zur Zukunft der Menschheit. Der Ich-Erzähler F.W. erscheint etwa hunderttausend Jahre nach seinem Ableben in der ,astromentalen Epoche“, die der gegenwärtigen „primitiven Zeit“ als „fortgeschrittene Zeit“ gegenübergestellt wird. (Werfel 1992 [1946], 34) Auf der einen Seite stehen die „astromentalen“ Menschen, bei denen die „Abkehr von der Natur“ vollkommen sei. (100) Zwar verzichten sie nicht auf Nachkommen, aber die Bevölkerung sei auf „jenes Minimum“ geschrumpft, das für den „Fortbestand einer Spezies unumgänglich notwendig“ sei. (53) Durchgehalten werde das „Einkindsystem“ jedoch nur von der „verfeinertsten Klasse“, die „kinderreichen Familien“ bildeten dafür die unterste Klasse der Gesellschaft. (54) Auf der anderen Seite hat sich eine Gruppe von den „Mentalen“ abgesetzt, sie bewohnen den sogenannten „Dschungel“ und widersetzen sich der zunehmenden Vergeistigung. Sie betreiben Ackerbau, setzen sich der Sonne aus, tragen grobe Kleidung und lassen ihre Haare wachsen - sie arbeiten und zeugen Kinder. Es kommt zu einem kurzen, extrem zerstörerischen Krieg, aber darum geht es in Werfels Roman nicht. Vielmehr subvertiert der Roman mit dieser Gegenüberstellung die Vorstellung eines unaufhörlichen Fortschritts. Denn der „Dschungel“ erscheint zwar als Rückfall der Natur in einen „vergangenen Zustand der Erdgeschichte“ (444), aber es besteht kein Zweifel darüber, so der Ich-Erzähler, dass sich beide in derselben „Geschichtszeit“ befanden. (518) Werfel geht es hier nicht darum, dass die Geschichte einen „Rückfall“ erleide, oder dass rohe Gewalt den Fortschritt vernichtet - zumal die astromentale Kultur ohnehin keine Zukunft mehr habe -, sondern es sind die „Widersprüche“ dieser „zukünftigen Erde“ (527), die den Erzähler beunruhigen und die Idee, Zukunft als eine ,bessere‘ Gegenwart zu entwerfen, in Frage stellen.

Die gesamte Erzählkonstruktion des zwischen 1943 und 1945 verfassten Romans ist als eine Reflexion über Zukunftswissen angelegt. ${ }^{9}$ Im ersten Kapitel

8 Vgl. dazu den Beitrag „Unordnung am Unort. Utopie und Diaspora in Franz Werfels Stern der Ungeborenen" von Roland Innerhofer in diesem Band.

9 Das hat die Forschung auch immer wieder hervorgehoben, folgende Beispiele seien hier genannt: Doberstein (1995) hebt den Aspekt der Utopiereflexion in dem Roman hervor, nach Strelka (1992, 180) machen Paradoxien die Grundstruktur der Existenzdarstellung aus, und Rode $(2002,14)$ geht in ihrer Monographie u.a. dem „Sammelsurium stilistischer und formaler Traditionen" nach. 
verteidigt sich der Erzähler gegenüber dem Vorwurf, er habe nicht über die Gegenwart geschrieben, über das „Brausen der Bomber“ und das „letzte Aufstöhnen“ der Millionen, die entehrt, gefoltert und massakriert wurden, wobei er doch selbst Opfer und Verfolgter sei. Nicht nur fand er keine geeignete Feder, gerade als er eine suchte, widerfuhr ihm die „Aussendung auf eine Forschungsreise“, über die er nun berichtet. (20-21) Werfels Ausrichtung auf die Zukunft ist weder als Flucht vor der Gegenwart noch als einfache Übertragung gegenwärtiger Ereignisse in die Zukunft zu begreifen. Vielmehr reflektiert der Roman sowohl die historischen Bedingungen des Nationalsozialismus als auch eine mögliche Nachkriegs-Zukunft, die Werfel, der 1945 im Exil starb, allerdings nicht mehr erlebte: nämlich die Konfrontation von Fortschritt einerseits und Rückfall in einen Naturzustand andererseits oder Themen wie den Krieg in der Zukunft, faschistische Gruppierungen oder das Fortbestehen von Christentum und Judentum.

Nicht alleine um einen Zukunftsentwurf geht es dem Roman somit, sondern um die Frage nach den Möglichkeiten, Zukunft zu entwerfen. Um ein „Historiker der Zukunft“ (19) zu werden, gibt es laut dem Erzähler nur zwei Wege: wissenschaftliche Folgerung und Traumdeuterei oder Wahrsagerei. Ersteres schließe sich selbst aus, da es die Wissenschaft höchstens zur Wahrscheinlichkeitsrechnung brächte. Dagegen verwiesen Traumdeuterei und Wahrsagerei auf eine „uralte Praxis“: „Die prophetischen Erkenntnisarten müssen es nur verstehen, um echt zu sein, die Schleier des Gleichnisses zu tragen und die Schatten des Geheimnisses zu werfen.“ (19) Auf dieser Grundlage führt der Erzähler das Paradox aus, als Prophet über eine „Forschungsreise“ als Historiker zu berichten, über die Zukunft also in der Vergangenheitsform zu schreiben. (58) Dass ihm hierbei nicht unbedingt „solide Tatsachen“ zur Verfügung stehen, ist keineswegs dem Bezug auf die Zukunft geschuldet: Der Historiker sei nämlich seit jeher ein „rückwärtsgewandter Prophet“, denn die Vergangenheit sei ebenso unwirklich wie die Zukunft (223), womit sich Werfels Roman in die Rezeptionsgeschichte von Friedrich Schlegels berühmtem Athenäums-Fragment $(1997,85)$ einreiht.

Als ein erzählender Prophet kann sich der Erzähler als Forscher und Historiker inszenieren und diese beiden Positionen zugleich immer wieder ironisch brechen, nicht zuletzt auch durch die Differenz zwischen dem erlebenden Ich, das sich in der Zukunft befindet, und dem erzählenden Ich, das aus der Zukunft in die Gegenwart zurückgekehrt ist. ${ }^{10}$ So ermahnt sich der Erzähler, ein Forschungsreisender solle schauen und schweigen, jedoch Interjektionen und Kritik ver-

10 Huber (1992) unterscheidet drei Instanzen: Erstens den verstorbenen F.W., zweitens den F.W. in der Zukunft (das erlebende Ich) und drittens den F.W., der aus der Zukunft in seine Gegenwart zurückgekehrt ist und den Reisebericht verfasst (erzählendes Ich). 
meiden. (Vgl. 135) Genau das tut er aber, er verhöhnt und verspottet seinen Begleiter, mischt sich in die laufenden Ereignisse ein und kritisiert seine Umwelt. Aber auch die Art und Weise, in der er seinen Bericht wiedergibt, thematisiert er: Das Hauptgeschäft eines Reiseschriftstellers, den er hier vorstelle, sei „Beschreiben und immer wieder Beschreiben“ und nicht Handlung, Verstrickung, Intrige und Lösung des Konflikts. (177) Er müsse das ihm „verhaßte Genre der Beschreibung“ aber auch wählen, weil über die zukünftige Welt keine Karten und Nachschlagewerke existierten: „Ein Bild ist nur dann verständlich, wenn es ein Nachbild von Vorbildern ist. Habe ich aber ein Vorbild?“ (178) Einerseits hebt er hier das Problem jedes Zukunftsromans hervor, andererseits bricht er diese Form doppelt: Erstens verweist er auf die Langweiligkeit einer ausführlichen beschreibenden Darstellung, in der viele Utopien verfasst seien, zweitens wählt er selbst gerade nicht eine solche Form: Sein Reisebericht enthält unzählige Intrigen, Konflikte, Wendungen und affektive Reaktionen auf die für ihn so unbekannten Welt.

Werfels Roman entfaltet sich entlang eines erzählerischen Dilemmas. Als Forschungsreisender und Historiker möchte er neutral die Wahrheit über alles berichten, der Bericht verselbständigt sich aber immer wieder, weshalb er von einem „völlig unkontrollierbaren Bericht“ spricht. (549, vgl. auch 634) Damit thematisiert er ,Zukunft' nicht nur, indem er ein enormes Spektrum an Formen des Zukunftswissens ausbreitet, sondern indem er seinen Erzähler die Problematik des Erzählens von Zukunft reflektieren lässt.

Was dabei stets bleibt, ist der Wille zum Erzählen und zum Erinnern. Denn gegen kaum etwas wehrt sich der Ich-Erzähler mehr als gegen den „See des Vergessens“ (640-650) und den „retrogenetischen Humus“, durch den die Menschen, die meinen, ihre Lebenszeit sei vorüber, sich über das Embryonal-Stadium zu Blastocysten rückentwickeln, die dann als Margeriten gepflanzt werden. (602614) Diese Verwendung menschlicher Überreste als ,Dünger' knüpft nicht nur an agrarwissenschaftliche Kreislauftheorien des 19. Jahrhunderts an: Die Nationalsozialisten verwendeten die Leichen-Asche ermordeter Juden tatsächlich zur Dünger-Herstellung, wie bereits der erste Dokumentarfilm über Konzentrationslager, Billy Wilders Death Mills (1945), zeigte. Damit verweist der Roman hier implizit auf das erste Kapitel zurück. Denn gegen das Vergessen der Schrecken des Krieges und des nationalsozialistischen Terrors instituiert sich der Erzähler gegen sämtliche Widerstände als ein unermüdlicher Berichterstatter, der verkündet, „was meine Augen sahen, meine Ohren hörten und mein Geist begriff“. (634) 


\section{Sememische Chronologie: Alban Nicolai Herbsts Anderswelt-Trilogie}

Die Selbstreflexion des Erzählens wird in Alban Nicolai Herbsts postmoderner Anderswelt-Trilogie ins Extreme getrieben. Die drei Romane - Thetis. Anderswelt (1998), Buenos Aires. Anderswelt (2001) und Argo. Anderswelt (2013) - entfalten ein immer wieder verwirrendes Erzähllabyrinth: ${ }^{11}$ Es finden sich unterschiedliche ineinander verflochtene Handlungsstränge mit einer Fülle von Figuren, Ortswechseln und Zeitsprüngen, ein Spiel mit Identitäten (realen Personen, dem Autor und seinen verschiedenen Erscheinungsformen, Figuren aus früheren Romanen usw.), verschiedene intertextuelle Bezüge und Verknüpfungen religiöser, mythologischer, technischer und naturwissenschaftlicher Metaphern und Erzählmuster. Zum Grundarrangement der Trilogie gehört der Wechsel zwischen der Gegenwart - ein wichtiger Bezugspunkt ist Berlin - und der Anderswelt, bis die Figuren selbst nicht mehr zwischen (ihrer) Realität, Traum, Simulation, Einbildung und Fiktion unterscheiden können.

Dabei kommt dem Spiel mit der Zeit eine wichtige Rolle zu, wie sich schon am Status der Anderswelt zeigt. So heißt es in Thetis über die Stadt, in der die Handlung spielt: „Trotzdem: Eine Zukunftsstadt war das nicht. Sondern eine andere Gegenwart.“ (Herbst 1998, 895). Ob die Anderswelt ein anderer Ort, eine Einbildung des Protagonisten Deters, eine auf einer CD-ROM gespeicherte Simulation ist oder doch in der Zukunft spielt, bleibt bis zum Ende unentschieden. Damit lassen sich die Romane nicht einfach Gattungen wie der Utopie oder Science Fiction zuordnen, auch wenn sie typische Zukunfts-Innovationen aufweisen. Es gibt zwar Holomorphe - computergenerierte Roboter mit Selbstbewusstsein -, die beginnen, ein Eigenleben anzunehmen, neue biologische Waffen, die menschliche Körper zersetzen, oder ein ,energetisches Kraftfeld“ namens Hodna (Herbst 1998, 43), aber vor allem kombinieren die Romane verschiedene semantische Felder, wie folgendes Beispiel verdeutlicht:

Bei Katerini strömten kleingefleckte Ameisen und dreißig Zentimeter lange schwarze Tausendfüßler vom Parnaß. Sie kamen, sie sprangen daher wie ein Bach, wälzten sich schon wie ein Fluß und die Flut hatte genügend Gift, Bullen zu töten. Im Hof der Reifenfabrik wieherten die Menschen, als ihnen Ameisen und Tausendfüßler die Beine hochkrabbelten, innen zwischen Hosenstoff und Haut. (Herbst 1998, 36)

11 Zu Herbsts postmodernem Erzählen vgl. z.B. Kühlmann 2003; Jürgensen 2007. 
Herbst verbindet das christlich-apokalyptische Bild der unzähligen, wie eine Flut über die Menschen herfallenden Tiere mit dem griechischen Topos Parnass, also dem Ort, der dem Gott Apollo und damit der Kunst geweiht ist. Die Reifenfabrik erscheint dann regelrecht als Bildbruch, erinnert aber gemeinsam mit den wiehernden Menschen an Tier-Horror-Filme wie etwa Formicula (1954).

Für den vorliegenden Zusammenhang ist aber vor allem wichtig, dass Herbsts Trilogie die Herstellung von Zukunft selbst thematisiert. Zum Beispiel ist die utopische Insel Levkas für einige Protagonisten das Ziel all ihrer Bestrebungen. Ob diese Insel aber wirklich existiert oder ob sie ein bloßes Wunschbild ist, lässt sich auch nach Lektüre der 2038 Seiten nicht abschließend entscheiden. Ein anderes Beispiel ist die Inszenierung von Prophezeiungen. In Thetis. Anderswelt wird der Aufstand des Ostens auf der Basis der Legende eines „Heiligen Kindes“ geführt, das die sogenannten Schänder opfern wollen, damit es von Thetis die Unsterblichkeit erhalte, zurückkehre und die „Europäische Stadt“ auslösche. (Herbst 1998, 461) Daher gebärt die Mongolin, Anführerin einer Widerstandsgruppe von Frauen, ein Kind, und Odysseus, Organisator des Widerstands, instrumentalisiert dieses Kind, um die Schänder-Heere zu organisieren. Der erste Band der Trilogie liefert somit eine Analyse der Funktionsweise von Prophezeiungen, indem er aufzeigt, welche Erwartungen und Ängste sie auslösen und formen, wie sie Handlungen organisieren und wie sie strategisch eingesetzt werden. Das gilt aber auch für die wissenschaftliche Prognose. So versucht die Geheimpolizei, zukünftige Aktionen terroristischer Gruppen vorherzusagen, was ebenso ins Leere läuft wie die Prophezeiungen der Sekten. Aber dennoch werden die Handlungsfelder der Akteure durch solche Prognosen und Prophezeiungen strukturiert und organisiert.

Wie dieser erzählerische Einsatz der Prophezeiung zeigt, ist die Zukunft bzw. die Zeit nicht einfach Gegenstand der Romane. Vielmehr sind Vor- und Rückverweise, Zeitsprünge und Zeitraffungen zentrale Strukturmerkmale, weshalb der Roman nicht einfach chronologisch aufeinander folgende und kausal zusammenhänge Ereignisse in einer anderen Reihenfolge präsentiert, sondern eine völlig andere Zeitlogik einführt.

Der erste Teil des Romans Thetis. Anderswelt setzt - nach einem Vorspiel ein mit der apokalyptischen Beschreibung einer sogenannten „geologischen Revision“ (Herbst 1998, 35), durch welche sich die Grundordnung der Welt in geologischer, geographischer und politischer Hinsicht ändert. Dabei stellt er zum einen die erdgeschichtliche Wende im Sinne von Erzählzeit und erzählter Zeit im Zeitraffer dar: Sie dauert einhundert Jahre ${ }^{12}$ und wird auf 16 Seiten dargestellt.

12 „Die Erdgeschichte zog sich in einhundert Jahren zusammen.“(Herbst 1998, 35) 
Dagegen finden sich die Protagonisten im zweiten Teil Buenos Aires immer wieder an einem bestimmten Tag, dem 1. November ein- und desselben Jahres wieder, obgleich die Zeit ständig vergeht. Zum anderen spielt das Vorspiel nicht nur auf kommende, erst noch zu erzählende Ereignisse an, sondern informiert auch über deren Ausgang. Zum Beispiel erfährt man schon hier, dass Sicherheitsbehörden die Myrmidonen, eine Terroristengruppe, zerschlagen werden, während die folgenden Kapitel die Entstehung dieser Gruppe beschreiben. Diesen Umgang mit Zeit thematisieren die Romane auch selbst: „Augenblicke sind Jahre, und was lang anmutet, ist in Wirklichkeit schnell vorbei. Und überhaupt die Zeiten! Die Chronologie ist nicht kausal, sondern sememisch [...]. Die Zeiten schließen aneinander, wo sie einander ausschließen müssen.“ (Herbst 1998, 506)

Die Zeit, das heißt Formen der Herstellung, Repräsentation und Manipulation von Zeit werden solchermaßen selbst zum Gegenstand der Romane. Zukunft kann daher in der Anderswelt-Trilogie nicht als einfache Folge der Gegenwart verstanden werden, sondern Zukunft ist in den Plural zu setzen - statt von der Zukunft ist von offenen Zukünften zu sprechen.

Für Herbst handelt es sich hierbei nicht einfach um eine literarische Technik der Ana- und Prolepsen, sondern um ein poetologisches Programm. In seinen Heidelberger Poetik-Vorlesungen hat er das nicht-lineare Erzählen in Spiegelungen und Wechselwirkungen, in Zyklen und Sprüngen als ökologisches Verfahren beschrieben, das aus einem kybernetischen Realismus folge. (Vgl. Herbst 2008, Kap. 3) Werke eines kybernetischen Realismus seien unabgeschlossen sowie unabschließbar und ihre Grundstruktur Regelkreise. Daher tendierten solche Werke zu Zyklen, die aber im Verhältnis zueinander „springen“ (Herbst 2008, 81), weshalb er lieber von „Regelspiralen“ spreche, welche jegliche Form der Linearität unterlaufen, ohne Zeitschlaufen zu bilden. Denn jede Wiederkehr zum Anfang trage die „Erfahrung des bislang durchlaufenen zyklischen Weges in sich“. (Herbst 2008, 81) Die offene und plurale Zukünftigkeit der Anderswelt-Trilogie findet hier ihre poetologische Begründung. Ganz in diesem Sinn endet der dritte Band Argo. Anderswelt mit den Worten: „[W]eil es doch nichts als rein Rausch war, worin ich - halb zu Hause, doch halb noch an Bord meines segelnden Schiffs - mit Freunden die menschliche Suche nach Zukunft menschlich aufs Meer trug.“ (Herbst 2013, 872)

\section{Schluss}

Nach Niklas Luhmann hat in der Neuzeit die Differenz von Vergangenheit und Zukunft die Führung der Zeitsemantik übernommen, da diese Unterscheidung am besten Zeitvorstellungen mit der Transformation von Gesellschaftsstrukturen in 
Einklang bringe. (Vgl. Luhmann 2003 [1991], 46) In diesem Kontext ist auch die Verzeitlichung der Utopie im 18. Jahrhundert zu verorten. Allerdings folgen Utopien diesem Schema nicht einfach, sondern thematisieren die Herstellung der Differenz von Vergangenheit und Zukunft, unterschiedliche Semantiken und Praktiken der Vorhersage sowie die Beziehungen zwischen Zeitvorstellungen und Gesellschaftsstrukturen. Weil sich Utopien daher seit dem 18. Jahrhundert an der für moderne Gesellschaften grundlegenden Zeitsemantik abarbeiten, das heißt vor allem am Basis-Narrativ ,Fortschritt‘, eröffnen sie einen futurologischen Reflexionsraum, in dem Möglichkeiten und Gefahren, Risiken und Nebenfolgen von Modernisierungsprozessen und alternativen Gesellschaftsformen durchgespielt werden können.

Die hier untersuchten Romane von Kellermann, Ettlinger, Werfel und Herbst zeigen das Spektrum unterschiedlicher Formen der Reflexion von Zukunftswissen auf - und zwar gerade entlang der Art und Weise, wie in der jeweiligen ZukunftsFiktion Zukunft verhandelt wird. Kellermanns Der Tunnel feiert nicht nur eine technokratische Vision, sondern fokussiert, wie ein Ingenieur zur Autorität in Hinsicht auf Zukunftsvorhersage und -handeln wird. In Ettlingers Der erschossene Storch erwacht eine Person nicht einfach einige Jahrtausende später, um Fortschritte beobachten zu können - wie etwa in Bellamys Looking Backward -, er stellt vielmehr die Frage, inwiefern die Menschheit nach einem furchtbaren Krieg überhaupt noch zukunftsfähig sei. Werfels Stern der Ungeborenen erzählt nicht nur von der Zukunft, sondern reflektiert die Erzählbarkeit von Zukunft. Und Herbsts Anderswelt-Trilogie macht Zukunft zum Material eines komplexen Erzählarrangements. Alle behandelten Romane reflektieren somit über die Zukunft ihre jeweilige Gegenwart und deren möglichen Zukunftsaussichten: Mit ,zukünftigem Zukunftswissen' erscheint sowohl die ,vergangene Gegenwart‘ (Jameson) als auch die gegenwärtigen Zukünfte und damit überhaupt die Vielfalt und Heterogenität des Futurischen: Formen, mit denen Zukunft vorhergesehen, vorausgesagt, errechnet, geplant, entworfen, erzählt, visualisiert oder angedeutet werden, Prognoseverfahren unterschiedlicher wissenschaftlicher Disziplinen, die Gleichzeitigkeit von religiösen, spirituellen und naturwissenschaftlichen Praktiken oder auch die politischen Dimensionen der Vorhersage. Damit sind Utopien literarische Erscheinungsformen einer kulturellen Prognostik.

\section{Literaturverzeichnis}

Artikel „Projectenmacher“. Grosses vollständiges Universal-Lexikon aller Wissenschaften und Künste, welche bishero durch menschlichen Verstand und Witz erfunden und verbessert worden. Bd. 29. Hg. Johann Heinrich Zedler. Leipzig u.a.: Zedler 1741, Sp. 784. 
Bühler, Benjamin, und Stefan Willer (Hg.). Futurologien. Ordnungen des Zukunftswissens. Paderborn: Fink, erscheint 2016.

Doberstein, Volker. „Die Unmöglichkeit des Möglichen. Utopie und Utopiekritik in Franz Werfels Exilroman ,Stern der Ungeborenen““. Literatur für Leser 18 (1995): 165-175.

Eco, Umberto. „Die Welten der Science Fiction“. Über Spiegel und andere Phänomene. München: DTV, 2002: 214-222.

Ettlinger, Karl. Der erschossene Storch. Leipzig: Goldmann, 1930.

Herbst, Alban Nicolai. Thetis. Anderswelt. Fantastischer Roman. Reinbek bei Hamburg: Rowohlt, 1998.

Herbst, Alban Nicolai. Buenos Aires. Anderswelt. Kybernetischer Roman. Berlin: Berlin Verlag, 2001.

Herbst, Alban Nicolai. Kybernetischer Realismus. Heidelberger Vorlesungen. Heidelberg: Manutius, 2008.

Herbst, Alban Nicolai. Argo. Anderswelt. Epischer Roman. Berlin: Elfenbein, 2013.

Herzl, Theodor. „Altneuland“ (1902). Wenn ihr wollt, ist es kein Märchen. Altneuland/Der Judenstaat. Hg. Julius Schoeps. Kronberg im Taunus: Jüdischer Verlag, 1978. 13-193.

Hock, Sabine. Karlchens Erfolg und Verfolgung. Die Lebensgeschichte des Schriftstellers Karl Ettlinger (1882-1939). Ettlinger Lesebuch 3. Nidderau: Naumann, 1997.

Hock, Sabine. „Ich bin ein Spötter nur, ein loser ... “. Karl Ettlinger (1882-1939) und seine Mitarbeit bei der Münchner Wochenschrift „Jugend“ in der Zeit von 1902 bis zum Beginn des Ersten Weltkriegs. Nidderau: Naumann, 1998.

Horn, Eva. Zukunft als Katastrophe. Frankfurt a.M.: Fischer 2014.

Huber, Lothar. „Der Erzähler und die Zeit in Franz Werfels Zukunftsroman ,Stern der Ungeborenen““. Unser Fahrplan geht von Stern zu Stern. Zu Franz Werfels Stellung und Werk. Hg. Joseph Strelka. Bern u.a.: Peter Lang, 1992. 219-230.

Huxley, Aldous. Brave New World (1932). London: Grafton, 1977.

Jameson, Fredric. „Progress Versus Utopia; Or, Can We Imagine the Future?“ Science Fiction Studies 9.2 (1982): 147-158.

Jürgensen, Christoph. „Ich sind auch andere. Zur Pluralisierung des Selbst in der Erzählprosa von Alban Nicolai Herbst“. Moderne. Postmoderne - und was noch? Hg. Ivar Sagmo. Bern u.a.: Peter Lang, 2007. 145-159.

Kellermann, Bernhard. Der Tunnel (1913). Stuttgart: Verlag Das Beste, 1985.

Koselleck, Reinhart. „Die Verzeitlichung der Utopie“. Utopieforschung. Interdisziplinäre Studien zur neuzeitlichen Utopie. Hg. Wilhelm Voßkamp. Bd. 3. Stuttgart: J.B. Metzler, 1982. $1-14$.

Krajewski, Markus (Hg.). Projektemacher. Zur Produktion von Wissen in der Vorform des Scheiterns. Berlin: Kadmos, 2004.

Kühlmann, Wilhelm. „Postmoderne Phantasien - Zum mythologischen Schreiben im Prosawerk von Alban Nicolai Herbst“. Euphorion 97 (2003): 499-516.

Leucht, Robert. „Die Figur des Ingenieurs im Kontext. Utopien und Utopiedebatten im ersten Drittel des 20. Jahrhunderts“. Internationales Archiv für Sozialgeschichte der Literatur 36 (2011): 283-312.

Lodge, David. „Imagining the Future (George Orwell)“. The Art of Fiction. Illustrated from Classic and Modern Texts. London: Penguin, 1992. 134-137.

Luhmann, Niklas. Soziologie des Risikos (1991). Berlin u.a.: De Gruyter, 2003.

Macho, Thomas, und Annette Wunschel (Hg.). Science \& Fiction. Über Gedankenexperimente in Wissenschaft, Philosophie und Literatur. Frankfurt a.M.: Fischer 2004. 
Rode, Silvia. Franz Werfels „Stern der Ungeborenen“. Die Utopie als fiktionaler Gegendiskurs und Ideengeschichte. Stuttgart: Heinz, 2002.

Schlegel, Friedrich. Kritische und theoretische Schriften. Stuttgart: Reclam, 1997.

Segeberg, Harro. Literarische Technik-Bilder. Studien zum Verhältnis von Technik- und Literaturgeschichte im 19. und frühen 20. Jahrhundert. Tübingen: Niemeyer, 1987.

Siebenpfeiffer, Hania. „Science Fiction“. Futurologien. Ordnungen des Zukunftswissens. Hg. Benjamin Bühler und Stefan Willer. Paderborn: Fink, erscheint 2016.

Strelka, Joseph. „Die politischen, sozialen und religiösen Utopien in Franz Werfels, Stern der Ungeborenen““. Franz Werfel im Exil. Hg. Wolfgang Nehring und Hans Wagener. Bonn: Bouvier, 1992. 175-186.

Suvin, Darko. Poetik der Science Fiction. Zur Theorie einer literarischen Gattung. Frankfurt a.M.: Suhrkamp, 1979.

Vieira, Fátima. „The Concept of Utopia“. The Cambridge Companion to Utopian Literature. Hg. Gregory Claeys. Cambridge: Cambridge University Press, 2010. 3-27.

Werfel, Franz. Stern der Ungeborenen. Ein Reiseroman (1946). Frankfurt a.M.: Fischer, 1992.

Willer, Stefan. „Vom Nicht-Wissen der Zukunft. Prognostik und Literatur um 1800 und um 1900“. Literatur und Nicht-Wissen. Historische Konstellationen in Literatur und Wissenschaft, 1750-1930. Hg. Michael Bies und Michael Gamper. Zürich, Berlin: Diaphanes, 2012. 171-196. 